\title{
Nanoscale Coordination Polymer Nanoparticles CPI-100
}

National Cancer Institute

\section{Source}

National Cancer Institute. Nanoscale Coordination Polymer Nanoparticles CPI-100. NCI

Thesaurus. Code C157499.

A preparation of self-assembled core-shell nanoscale coordination polymer (NCP)

nanoparticles containing an as of yet undisclosed payload with potential

immunostimulating and antineoplastic activities. Upon intravenous administration, NCP

nanoparticle formulation CPI-100 delivers its payload to tumor cells, which may lead to

enhanced immune-mediated killing and regression of tumor cells. 